\title{
Positronium lifetime in polymers
}

\author{
Abel Camachd* \\ Department of Physics \\ Universidad Autónoma Metropolitana-Iztapalapa \\ Apartado Postal 55-534, \\ C.P. 09340, México, D.F., México.
}

(Dated: November 16, 2018)

\begin{abstract}
A model describing the relationship between the ortho-positronium lifetime and the volume of a void, located in a synthetic zeolite, is analyzed. Our idea, which allows us to take into account the effects of temperature, comprises the introduction of a non-hermitian term in the Hamiltonian, which accounts for the annihilation of the ortho-positronium. The predictions of the present model are also confronted against an already known experimental result.
\end{abstract}

Key words: Positronium lifetime, polymers

\section{INTRODUCTION}

In the context of polymer research one of the most interesting issues is connected with the relationship between molecular relaxation and free-volume properties 1]. Experimentally several techniques have been already developed in order to determine the size of these voids in polymers, for instance, small-angle $\mathrm{X}$ ray diffraction [2], neutron diffraction [3], or positron annihilation spectroscopy [4], in which the positron or positronium play the role of a probe, and the lifetimes of these systems are monitored.

The current experimental results give evidence that the positron and positronium are localized in the preexisting free--volumes in polymers [5], and in consequence one could relate the size of the free--volume region with the detected lifetime [6].

The use of positron or positronium lifetimes to determine the size of voids faces severe difficulties [1], which leave only the possibility of employing approximated approaches to determine the correlation between lifetime and free-volume. Clearly this is an indirect method, and in consequence we need a model to link the measured parameter with the required variable. Usually this requirement has been satisfied employing the so called standard model [7]. Nevertheless it must be underlined that, as already noted [7], this model embraces several deficiencies.

In the present work it will be assumed that the positronium is located in a spherical wall, but, in order to avoid one of the existing drawbacks (to wit, that the matrix electrons form a layer of homogeneous density and thickness), we will not only consider a finite wall, but also we will introduce a non-hermitian term in the Hamiltonian, which accounts then for the annihilation of the positronium due to its interaction with the polymer. In this way the atomic structure of the polymer is taken into account in a more realistic manner, though, of course, the description of this interaction can be improved. Additionally we

*Electronic address: acq@xanum.uam.mx may consider not only the ground state of the particle (as happens in the standard model), but also excited states, in other words, our model does not necessarily imply a low temperature.

Our final result will be the radius of the void in the form of a polynomial, in which the coefficients will be related to variables that could be detected in an experiment, see (9) below, where the radius of the void, $R$, does define indeed a polynomial, in which the coefficients are functions of parameters that are under control in an experiment, for instance, $\tau$ (lifetime), $m$ (mass of the particle), etc.

\section{NON-HERMITIAN POTENTIALS}

As usual in the standard model, we treat the orthopositronium as a single particle, and refer its wave function with respect to its center of mass. As mentioned above, we analyze the relationship between the orthopositronium lifetime and a spherical free-volume region with the introduction of a non-hermitian term in the potential associated with the barrier. Hence we have that the potential to consider reads

$$
\begin{gathered}
V(r)=0, \quad r<R, \\
V(r)=V_{0}+i \gamma, \quad r \geq R .
\end{gathered}
$$

Here $\gamma \leq 0$ and $V_{0} \geq 0$. Comparing with the standard model [7] we may see that in our case we do not assume a certain thickness $\Delta R$ associated with the layer defined by the matrix electrons. At this point it is noteworthy to mention that this assumption of the standard model is used also to calculate the wave function in such a way that the corresponding Schrödinger equation has the same form inside the hole and in the region inside the 
polymer defined by $R \leq r \leq R+\Delta R$ [7], a questionable fact, since this requirement neglects the potential in the aforementioned region. Our approach does not need this kind of approximation.

We may fathom deeper the introduction of the imaginary term in the potential noting that in the standard model (see, for instance, 1]) a homogeneous electron layer with a thickness $\Delta R$ must be introduced. Inside this layer takes place the annihilation process, in other words, it is a relevant parameter of the model. Clearly, $\Delta R$ is an empirical variable, and though in the present model we have, at this level, also an empirical parameter, namely, $\gamma$, there are some important differences between the two aforementioned approaches that must be underlined. Indeed, for instance, in the standard model (see figure 1 of [1]) the wave function of the positronium (which is, strictly, valid only inside the hole) is extrapolated to the electron layer. It is readily seen that in this case the distortion of the wave function due to the presence of the layer is not taken into account. In addition, since quantization emerges from the introduction of boundary conditions, it is also clear that the thickness of the layer, in the standard model, does not impinge upon the quantization of some of the variables of the positronium, i.e., the energy.

In our proposal the rate of annihilation is considered as an empirical parameter, $\gamma$, (this statement can be understood better remembering that in the probability conservation equation $\gamma$ plays the role of a sink, see (6) below), and hence we do not need to extrapolate the wave function to any region not contained in the hole (therefore we do not change the quantization of the energy, for instance). In other words, we reduce, compared to the standard model, the number of assumptions.

Additionally, the condition of an infinite potential barrier is not imposed, as is usual in some previous approaches [1, 7]. The presence of the matrix of electrons is considered with introduction of the extra term, the non-hermitian contribution in the potential energy, and it will play the role of a sink for our particles.

Performing the usual procedure we have that the radial part of the wave function has two different expressions in terms of spherical Bessel functions

$$
\begin{gathered}
y_{l}(r)=A j_{l}(\rho), \quad r<R, \\
y_{l}(r)=B j_{l}(\tilde{\rho})+C \eta_{l}(\tilde{\rho}), \quad r \geq R .
\end{gathered}
$$

Here we have that $\rho=\sqrt{\frac{2 m E_{l}}{\hbar^{2}}} r$, and $\tilde{\rho}=$ $\sqrt{\frac{2 m\left(E_{l}-V_{0}-i \gamma\right)}{\hbar^{2}}} r$.

Quantization of energy appears when we consider the continuity conditions upon these two expressions, and also upon their logarithmic derivatives on the surface $r=$ $R$. Clearly, it is possible to consider the case in which the involved particle is in an excited state $(l \neq 0)$. In other words, this approach allows us to consider the possibility of taking into account, at least partially, the effects of temperature.

If $l=0$, then the energy reads (approximately)

$$
E_{0}=V_{0}+\sqrt{\frac{\hbar^{4} \Omega^{2}}{4 m^{2} R^{4}}-\gamma^{2}} .
$$

The parameter $\Omega$, a consequence of the continuity conditions, is equal to 5.15. Clearly we may express the microscopic parameter $\gamma$ as a function of $E_{0}, R$, and $V_{0}$.

\section{ORTHO-POSITRONIUM LIFETIME AND FREE-VOLUME RADIUS}

At this point a link between lifetime and the solutions to the Schrödinger is needed, here we assume that the measured lifetime comprises two different terms:

1) The time that a particle, with energy $E_{0}$ and mass $m$, needs to travel a distance equal to the radius of the free-volume region.

2) The decay time of an ortho-positronium located inside the polymer.

Concerning these two contributions to the lifetime it has to be mentioned that in the first part we have a semiclassical approach, which means that several conditions have to be fulfilled in order to obtain a good approximation, for instance, the de Broglie wavelength of the particle, $\lambda_{c}=\frac{\hbar}{p}$, has to be smaller than the radius, i.e., $\lambda_{c}<R$.

The second part of the present model considers the fact that the annihilation process takes place inside the polymer at a distance from the surface $r=R$, which is negligible compared with $R$.

If we consider the continuity equation at any point inside the polymer we find that

$$
\frac{\partial \hat{\rho}}{\partial t}+\nabla \cdot \mathbf{J}=\frac{2 \gamma}{\hbar} \hat{\rho} .
$$

Here $\hat{\rho}$ denotes the probability density associated with the ortho-positronium and $\mathbf{J}$ the corresponding probability current. At a fixed point inside the material we have that

$$
\hat{\rho}=\exp \left(\frac{2 \gamma}{\hbar} t\right) f(\vec{r})
$$


This last expression $(f(\vec{r})$ is a function of the position) clearly shows that $\gamma$ is related to the decay of the ortho-positronium when it is not in the void, and in consequence we may link it with second part of our assumption. In this way we find that it renders a contribution to the lifetime that goes (approximately) like $\tau_{2}=-\frac{\hbar}{2 \gamma}$.

Hence the lifetime is given by

$$
\tau-\frac{\hbar}{2 \gamma}+\sqrt{\frac{m R^{2}}{2 E_{0}}}
$$

At this point it is noteworthy to comment that we have assumed, from square one, that the positronium is always localized inside the void. In other words, we do not consider a modification of a situation in which the positronium may escape from the hole, a case already studied [10].

\section{THEORETICAL PREDICTIONS}

It is readily seen that in our last expression, if we seek to obtain information concerning the size of the void, then we must know the terms which are available in an experiment. In some cases 9$]$ the annihilation rate inside the electron layer is an experimental result, in our case this means that we may know the value of $\gamma$. Bearing this in mind we may cast (5) and (8) as a polynomial equation for the radius

$$
\begin{gathered}
\frac{m^{4}}{\left(\tau+\frac{\hbar}{2 \gamma}\right)^{4}} R^{8}-4 \frac{m^{3} V_{0}}{\left(\tau+\frac{\hbar}{2 \gamma}\right)^{2}} R^{6} \\
+4 m^{2}\left(V_{0}^{2}+\gamma^{2}\right) R^{4}=\hbar^{4} \Omega^{2} .
\end{gathered}
$$

Let us now introduce the following definition

$$
R=\sqrt{\frac{4 \tau^{2} V_{0}}{m}} r .
$$

Then we may rephrase (9) in terms of the dimensionless parameter $r$

$$
r^{8}-r^{6}+\frac{1}{2} r^{4}=\frac{\hbar^{4} \Omega^{2}}{4^{4} \tau^{4} V_{0}^{4}} .
$$

This last expression embodies the main result in the present work. In order to resort to (9) as a model linking the lifetime and void size we require, in addition, the value of $\gamma$ and $V_{0}$. As previously mentioned, in some materials the annihilation rate inside the electron layer is known. Forsooth, in the case of positronium the annihilation rate inside the electron layer is the spin-averaged rate for the ortho-Ps and para-Ps, which reads $2 n s^{-1}$ 9]. In our case, this implies that

$$
\frac{1}{2} n s=-\frac{2 \gamma}{\hbar}
$$

It is readily seen that a theoretical prediction requires a deeper knowledge of the electron layer, to wit, the value of $V_{0}$. It is also clear that the evaluation of this parameter lies outside the realm of the present approach. Nevertheless, in order to have an idea of the possibilities of the present model let us now assume that $V_{0}$ has an order of magnitude similar to the contribution of one atom to the internal energy of a solid (within the Einstein model [? ]) in the limit in which the temperature of the solid is much higher than the characteristic Einstein temperature. Therefore

$$
V_{0}=\kappa T \text {. }
$$

The introduction now of $T \sim 300 K$, in the case of the lifetime of an $\alpha$-cage, of the so-called MS-4A zeolite [8], the one reads $\tau \sim 5.9 \mathrm{~ns}$, renders

$$
R=0.42 n m \text {. }
$$

The experimental value for this case reads [8]

$$
R=0.57 \mathrm{~nm} \text {. }
$$

In other words, our model provides a radius that has the same order of magnitude than the experimental measurement.

\section{CONCLUSIONS}

The case of an ortho-positronium immersed in a spherically symmetric potential wall, in which the potential barrier contains a non-hermitian term, has been considered as a model to obtain a correlation between lifetime and free-volume regions in some polymers.

This approach allows us to take into account the effects of temperature, i.e., we may consider in expressions (3) and (4) the case $l \neq 0$, a fact that implies that the particle is not in its ground state. Indeed, let us now suppose that $l=1$, then

$$
\begin{gathered}
y_{1}(r)=A\left[\frac{\sin (k r)}{(k r)^{2}}-\frac{\cos (k r)}{(k r)}\right], \quad r<R \\
y_{1}(r)=B\left[\frac{\sin (\tilde{k} r)}{(\tilde{k} r)^{2}}-\frac{\cos (\tilde{k} r)}{(\tilde{k} r)}\right] \\
+C\left[\frac{\cos (\tilde{k} r)}{(\tilde{k} r)^{2}}+\frac{\sin (\tilde{k} r)}{(\tilde{k} r)}\right], \quad r \geq R .
\end{gathered}
$$


Proceeding in the same way as in the first case we find that

$$
E_{1}=V_{0}+\sqrt{\frac{\hbar^{4} \hat{\Omega}^{2}}{4 m^{2} R^{4}}-\gamma^{2}} .
$$

The parameter $\hat{\Omega}$, a consequence of the continuity conditions, is equal to 17.49. In a first approximation we have that the present models predicts

$$
E_{1}-E_{0}=13 \frac{\hbar^{2} \pi^{2}}{m R^{2}} .
$$

In the standard model this energy difference [8] reads $\Delta E=3 \frac{\hbar^{2} \pi^{2}}{m R^{2}}$, in other words, our prediction is $13 / 3$ times larger than the one stemming from the standard model.

Clearly, one of the mayor objections to the standard model, spherical symmetry, is here also present, and, as it has already been pointed out [7], even ellipsoidal symmetry is unlikely to represent the real situation. Nevertheless, the simple case that we have considered here allows us to obtain analytical results (we do not have to resort to molecular dynamics simulations in order to improve the standard model [7]).

Additionally we avoid a second drawback of the standard model, closely related to the presence of a layer of homogeneous density, and thickness $\Delta R$, defined by the matrix electrons. Indeed, the introduction of this layer entails also an additional condition, to wit, the wave function inside the layer is taken from the solution that emerges inside the spherical hole [1, 7], a situation that can not be correct. In our case, we do not need introduce this assumption.

The effects of the polymer are taken into account with the introduction of $\gamma$, a microscopic parameter that can be straightforwardly connected with an experimental output [9], and with $V_{0}$. Resorting to a very rough assumption, expression (13), has rendered an order of magnitude for the radius of the void that matches with the experimental readout. Of course, a more precise prediction demands a more physical value for $V_{0}$ (than the one here employed), nevertheless (14) and (15) show that the present model could be a useful one.

To conclude, it is already a known result [12] that the holes show a significant asphericity. Hence, a further case in the context of the present work is related to the possibility of improving the standard model trying to generalize the present approach to situations lacking spherical symmetry, for instance, considering first ellipsoids, as in [1], and introducing non-hermitian terms in the potential energy. Though, as 1] clearly shows, we must resort to numerical methods.

\section{Acknowledgments}

This research was partially supported by CONACYT Grant 42191-F. The author would like to thank A.A. Cuevas-Sosa and A. Macías for useful discussions and literature hints.
[1] Y. C. Jean and H. Shi, Non. Crst. Sol. 172-174, 806 (1994).

[2] S. Nojima, R. J. Roe, D. Rigby, and C. C. Han, Macromol. 21, 4305 (1990).

[3] J. S. Royal, J. G. Victor, and J. M. Torkelson, Macromol. 25, 4702 (1992).

[4] W. C. Yu, C. S. P. Sung, and R. E. Robertson, Macromol. 21, 355 (1990).

[5] Q. Deng, C. S. Sundar, and Y. C. Jean, J. Phys. Chem. 96, 492 (1992).

[6] S. J. Tao, J. Chem. Phys. 56, 5499 (1972).
[7] H. Schmitz and F. Müller-Plathe, J. Chem. Phys. 112, 1040 (2000).

[8] Y. Ito, T. Takano, and M. Hasegawa, Appl. Phys. A45, 193 (1988).

[9] Y. C. Jean, Macromol. 29, 5756 (1996).

[10] Y. C. Jean, Microchem. J. 42, 72 (1990).

[11] G. Czycholl, Theoretische Festkörperphysik (Friedr. Vieweg und Sohns Verlag, Braunschweig 2000).

[12] Y. C. Jean, H, Nakanishi, L. Y. Hao, and T. C. Sandreczki, Phys. Rev. B42, 9705 (1990). 